\title{
Association of Hand Grip Strength and Cardiometabolic Markers in Korean Adult Population: The Korea National Health and Nutrition Examination Survey 2015-2016
}

Hanul Chong', Young Eun Choi ${ }^{2, *}$, Jin Young Kong' ${ }^{1}$ Joo Hyun Park', Hyun Jeong Yoo', Jeong Ho Byeon', Hye Jun Lee', Sang Hyun Lee ${ }^{2}$

'Department of Family Medicine, Yonsei University College of Medicine, Seoul, Korea

${ }^{2}$ Department of Family Medicine, National Health Insurance Service Ilsan Hospital, Goyang, Korea

\section{See editorial commentary page on 271}

Background: Muscle strength has been suggested as a cardiovascular marker. The aim of this study was to examine the associations between hand grip strength and biomarkers of cardiovascular disease in the Korean population. Methods: A total of 9,083 participants aged 20-80 years from Korea National Health and Nutrition Examination Survey 2015-2016 were investigated.

Results: Among men, both relative and dominant hand grip strength showed a positive association with diastolic blood pressure in those aged 65-80 years (95\% confidence interval, P-value of dominant and relative hand grip strength: $\beta=0.06,0.01 ; \mathrm{P}<0.05)$. Among women, relative and dominant hand grip strength showed a positive relationship to diastolic blood pressure in those aged $20-64$ years $(\beta=0.06,0.01 ; \mathrm{P}<0.001)$. Body mass index was positively associated with dominant hand grip strength in younger women $(\beta=0.18, P<0.05)$, whereas it was positively associated with relative hand grip strength in all sex and age groups. High-sensitivity C-reactive protein showed a negative association with relative and dominant hand grip strength in all women, although the same association was observed only in younger men. Diabetes was inversely related to hand grip strength in younger women and men.

Conclusion: Increased hand grip strength may be associated with lower C-reactive protein in women and with less risk of diabetes in the Korean adult population. Further prospective studies are needed for the determination of causality between cardiometabolic markers and hand grip strength.

Keywords: Hand Strength; Blood Pressure; Inflammation; Cross-Sectional Studies

Received: August 1, 2018, Revised: December 13, 2018, Accepted: December 27, 2018

${ }^{*}$ Corresponding Author: Young Eun Choi https://orcid.org/0000-0002-7657-7776

Tel: +82-31-900-0114, Fax: +82-31-900-0149, E-mail: yechoi@nhimc.or.kr 


\section{INTRODUCTION}

Hand grip strength (HGS) is a parameter used in diagnosing sarcopenia. Sarcopenia has been proven to be closely linked to future disability, frailty, metabolic syndrome, ${ }^{1)}$ diabetes mellitus, ${ }^{2)}$ and mortality. ${ }^{3)}$ Muscle strength is an important parameter in predicting health, and HGS is considered a representative marker of muscle strength. ${ }^{4)}$

Some studies have shown a link between HGS and inflammatory markers closely related to cardiovascular diseases. ${ }^{1,5)}$ High levels of high-sensitivity C-reactive protein (hsCRP) have been suggested to be related to low HGS. ${ }^{6,7)}$ In a study in US adults in 2016, higher relative HGS was significantly associated with lower systolic blood pressure, glucose, plasma insulin, and triglycerides, and with higher high-density lipoprotein (HDL) cholesterol. ${ }^{8)}$ In one recent study, HGS showed only a moderate relationship to cardiovascular markers. ${ }^{4}$ This result may be attributed to the use of dominant HGS. A study in Taiwan showed significant associations between relative HGS and favorable cardiometabolic risk factors such as blood pressure, triglyceride, total cholesterol-to-HDL ratio, uric acid, hemoglobin AlC (HbAlC), and Framingham score in men, and fasting glucose, $\mathrm{HbAlC}, \mathrm{HDL}$, and log hsCRP in women. ${ }^{9)}$ Another study in China also showed a relationship between higher relative HGS and favorable blood lipid profile and lower dyslipidemia risk in both men and women, whereas higher absolute HGS was associated with unfavorable metabolic profiles and higher metabolic disease risk. ${ }^{10)}$

Several studies on the Korean population have evaluated muscle strength by measuring HGS. In one study, sarcopenia was reported to be related to cardiovascular disease independent of other cardiovascular risk factors in elderly Korean adults. ${ }^{11)}$ Another study showed a negative association between metabolic syndrome and HGS divided by body weight only in elderly Korean men, with a stronger effect in those aged $>65$ years. ${ }^{12)}$ A cross-sectional study showed an inverse relationship between metabolic syndrome and relative HGS in adults. ${ }^{13)}$ A recent Korean study demonstrated a relationship between low HGS and decreased quality of life in the adult population. ${ }^{14)}$ In a study using data from the Korea National Health and Nutrition Examination Survey (KNHANES) 2014-2015, a multivariate logistic regression analysis of 8,208 participants showed that HGS was inversely associated with type 2 diabetes. The study also found that hsCRP mediated approximately $10 \%$ of the association between HGS and type 2 diabetes mellitus. ${ }^{15)}$ In this study, sex and old age were analyzed together despite a significantly different HGS between male and female participants (mean HGS, 39.67; 95\% confidence interval [CI], 39.32-40.02 for men and mean HGS, 23.70; 95\% CI, 23.48-23.91 for women aged between 19 and 80 years). Men and women are physiologically different, and HGS is two times higher in men than in women. Further, people become weaker with age. Therefore, we wanted to determine the differences among different sex and age groups. To our knowledge, no study has assessed the direct relationship between HGS and cardiometabolic markers in stratified age and sex groups. The purpose of this study was to examine the associations between types of HGS and biomark- ers of cardiovascular disease risk, including blood pressure, serum lipids, fasting plasma glucose, and hsCRP in the Korean adult population. Further, we aimed to examine the relationship between HGS and metabolic diseases including diabetes mellitus and dyslipidemia.

\section{METHODS}

\section{Participants}

Data were collected from KNHANES 2015-2016. KNHANES is a nationwide population-based cross-sectional survey that consists of health interviews, health examinations, and nutritional surveys. Data from January 1, 2015, to December 31, 2016, were collected. Of a total of 15,530 participants, those aged $20-80$ years $(n=12,170)$ were included in this study. Participants with missing data on HGS, laboratory tests, or blood pressure were excluded. Participants were also excluded if they presented any of the following conditions: pain or stiffness in the last 7 days; fracture or injury of the hands, fingers, or arms; paralysis of the hands; any cast or bandages applied on the hands and wrists; and any surgery in the last 3 months. Finally, a total of 9,083 participants were included in the analysis. Data were analyzed with a weighted ratio of 1:1 and a weighted total number of participants of $32,116,183$.

\section{Ethics Statement}

Individual patient consent was waived since this study was a retrospective analysis. The study was performed in agreement with the Declaration of Helsinki. ${ }^{16)}$

\section{Health Examination Survey}

A self-administered questionnaire with health examination items was individually completed by the participants, and collected to obtain information on age, sex, physical activity, and smoking and drinking status. Participants who were currently smoking and had smoked $>100$ cigarettes in their lifetime were considered smokers. Participants who had consumed more than one glass of alcohol in the recent year were considered drinkers. Activity levels were examined in minutes per day, by summing the durations of moderate to vigorous physical activities spent in work and leisure. The participants were asked to report if they had been diagnosed with diabetes and dyslipidemia, and if they had been treated for these diseases.

\section{Definition of Hand Grip Strength}

Dominant HGS was defined as the grip strength of the dominant hand measured in kilograms. ${ }^{17)}$ Absolute HGS was defined as the sum of the largest readings for both hands. Relative HGS was defined as absolute HGS divided by body mass index (BMI). ${ }^{18)}$

\section{Measurement of Hand Grip Strength}

HGS was measured using a digital hand dynamometer (T.K.K 5401 digital grip strength dynamometer; Takei Scientific Instruments Co. Ltd., Tokyo, Japan). Measurement was performed according to the 
Southampton protocol for adult grip strength measurement, as follows: ${ }^{17)}$ in a sitting position with a neutral position of the forearms rested on the arms of a chair. HGS was measured three times for both hands, and the maximum strength was selected for the analysis. A rest interval of at least 30 seconds was provided between measurements. If dominancy was not mentioned, the right hand was considered the dominant hand.

\section{Other Data}

Anthropometric measures including height, weight, and waist circumference were collected using a standardized procedure. BMI was calculated as weight $(\mathrm{kg})$ divided by height squared $\left(\mathrm{m}^{2}\right)$. Cardiometabolic markers such as fasting glucose, $\mathrm{HbAlC}$, systolic blood pressure, diastolic blood pressure, total cholesterol, triglyceride, HDL, low-density lipoprotein (LDL), and hsCRP were measured.

\section{Definition of Cardiometabolic Disease}

Participants with a fasting plasma glucose level of $\geq 126 \mathrm{mg} / \mathrm{dL}$ or with antidiabetic treatment were categorized into the diabetes group according to the definition of the American Diabetes Association. ${ }^{19)}$ Participants using lipid-lowering drugs were categorized into the dyslipidemia group, along with those with at least one of the following lipid profiles: total cholesterol $\geq 200 \mathrm{mg} / \mathrm{dL}, \mathrm{LDL} \geq 130 \mathrm{mg} / \mathrm{dL}$, and triglyceride $\geq 150 \mathrm{mg} / \mathrm{dL}$, in accordance with the definition in the guidelines of the American Association of Clinical Endocrinologists and American
College of Endocrinology.

\section{Statistical Analysis}

To verify the characteristics of the participants, we presented the frequency of categorical variables and the weighted percentage. The RaoScott chi-square test was performed for categorical variables. Continuous variables were analyzed with t-test or analysis of variance, and presented as means and standard errors (SEs).

To examine multicollinearity, the variance inflation factor was calculated. To handle the problem of multicollinearity, we eliminated variables with a variance inflation factor of $>10$. Age-adjusted univariate linear regression analysis was performed to examine the association between HGS and cardiometabolic variables. Multivariate linear regression analysis was conducted thereafter, with adjustment for age, smoking status, and drinking status.

As men and women had significantly different mean values for several characteristics, we stratified the participants by sex. To examine the difference in older age, we also stratified the participants by age (20-64 and 65-80 years). All analyses were conducted using the statistical package IBM SPSS ver. 23.0 for Windows (IBM Corp., Armonk, NY, USA). Statistical significance was considered at $\mathrm{P}<0.05$. Analysis was performed using a complex sample design.

Table 1. Comparison of demographics between male and female participants

\begin{tabular}{|c|c|c|c|c|}
\hline \multirow{2}{*}{ Characteristic } & \multicolumn{2}{|c|}{ Sex } & \multirow{2}{*}{ Total } & \multirow{2}{*}{ P-value } \\
\hline & Male & Female & & \\
\hline No. of participants & $4,062(51.0)$ & $5,021(49.0)$ & $9,083(100.0)$ & \\
\hline Age (y) & $45.62 \pm 0.32$ & $46.89 \pm 0.34$ & $46.24 \pm 0.28$ & $<0.001$ \\
\hline BMI $\left(\mathrm{kg} / \mathrm{m}^{2}\right)$ & $24.53 \pm 0.07$ & $23.31 \pm 0.07$ & $23.93 \pm 0.05$ & $<0.001$ \\
\hline Waist circumference (cm) & $86.28 \pm 0.18$ & $78.94 \pm 0.20$ & $82.68 \pm 0.14$ & $<0.001$ \\
\hline Systolic blood pressure (mm Hg) & $120.09 \pm 0.29$ & $114.54 \pm 0.32$ & $117.37 \pm 0.24$ & $<0.001$ \\
\hline Diastolic blood pressure $(\mathrm{mm} \mathrm{Hg})$ & $78.30 \pm 0.21$ & $73.22 \pm 0.18$ & $75.81 \pm 0.15$ & $<0.001$ \\
\hline Total cholesterol (mg/dL) & $191.94 \pm 0.66$ & $193.12 \pm 0.57$ & $192.52 \pm 0.47$ & 0.151 \\
\hline Triglycerides (mg/dL) & $168.26 \pm 3.33$ & $113.96 \pm 1.37$ & $141.66 \pm 1.91$ & $<0.001$ \\
\hline High-density lipoprotein (mg/dL) & $47.47 \pm 0.22$ & $55.14 \pm 0.23$ & $51.23 \pm 0.17$ & $<0.001$ \\
\hline Low-density lipoprotein (mg/dL) & $112.98 \pm 0.60$ & $115.48 \pm 0.50$ & $114.20 \pm 0.43$ & $<0.001$ \\
\hline Fasting glucose (mg/dL) & $102.02 \pm 0.48$ & $97.18 \pm 0.40$ & $99.65 \pm 0.33$ & $<0.001$ \\
\hline Hemoglobin A1c (\%) & $5.66 \pm 0.02$ & $5.59 \pm 0.01$ & $5.63 \pm 0.01$ & $<0.001$ \\
\hline High-sensitivity C-reactive protein (mg/L) & $1.32 \pm 0.04$ & $1.11 \pm 0.03$ & $1.21 \pm 0.03$ & $<0.001$ \\
\hline Physical activity (min/wk) & $45.85 \pm 2.12$ & $21.81 \pm 0.97$ & $34.08 \pm 1.24$ & $<0.001$ \\
\hline Dominant hand grip strength (kg) & $41.83 \pm 0.18$ & $24.91 \pm 0.11$ & $33.54 \pm 0.15$ & $<0.001$ \\
\hline Absolute hand grip strength (kg) & $81.69 \pm 0.33$ & $48.28 \pm 0.20$ & $65.32 \pm 0.28$ & $<0.001$ \\
\hline Relative hand grip strength $(\mathrm{kg} / \mathrm{BMl})^{*}$ & $3.37 \pm 0.01$ & $2.11 \pm 0.01$ & $2.75 \pm 0.01$ & $<0.001$ \\
\hline Diabetes & $582(10.8)$ & $505(8.6)$ & $1,087(9.7)$ & $<0.001$ \\
\hline Dyslipidemia & $2,624(64.1)$ & $2,999(56.0)$ & $5,623(60.1)$ & $<0.001$ \\
\hline Smoking & $1,390(37.6)$ & $238(5.4)$ & $1,628(21.8)$ & $<0.001$ \\
\hline Drinking & $2,937(75.2)$ & $2,072(44.7)$ & $5,009(60.3)$ & $<0.001$ \\
\hline
\end{tabular}

Values are presented as unweighted number (weighted \%) for categorical variables or mean \pm standard error for continuous variables. P-values are from the Rao-Scott $\chi^{2}$ test or t-test.

BMl, body mass index.

*Relative hand grip strength is defined as absolute hand grip strength divided by BMI. 


\section{RESULTS}

\section{Characteristics of the Participants}

The data of 9,083 participants were analyzed. The mean age of the participants was $46.24 \pm 0.28$ years. The mean values and SEs for cardiometabolic outcomes, grip strength, and BMI according to sex are shown in Table 1. The mean absolute HGS was $81.69 \pm 0.33$ in men and $48.28 \pm 0.20$ in women. The relative HGS was $3.37 \pm 0.01$ in men and $2.11 \pm 0.01$ in women. Men showed higher BMI, higher waist circumference, longer physical activity duration, and higher systolic and diastolic blood pressure. With respect to biomarker outcomes, men showed higher triglyceride, lower HDL, lower LDL, higher fasting plasma glucose, higher $\mathrm{HbAlC}$, and higher CRP levels. More men than women had diabetes and dyslipidemia (diabetes $10.8 \%$ versus $8.6 \%$, dyslipidemia $64 \%$ versus $56.0 \% ; \mathrm{P}<0.001$ ) (Table 1 ). Men tended to smoke and drink more than women (smokers $37.6 \%$ versus $5.4 \%$, drinkers $75.2 \%$ versus $44.7 \%$; $\mathrm{P}<0.001)$.

\section{Linear Regression Analysis}

Age-adjusted univariate regression analysis was performed to examine the association between HGS and age- and sex-stratified variables. To optimize multicollinearity, we eliminated total cholesterol, which

Table 2. Results from univariate linear regression analysis on the associations of relative handgrip strength (strength/BMl) and dominant handgrip strength (kg) with cardiometabolic markers

\begin{tabular}{|c|c|c|c|c|c|c|c|c|}
\hline \multirow{3}{*}{ Predictor } & \multicolumn{4}{|c|}{ Men } & \multicolumn{4}{|c|}{ Women } \\
\hline & \multicolumn{2}{|l|}{ Aged 20-64 y } & \multicolumn{2}{|l|}{ Aged 65-80 y } & \multicolumn{2}{|l|}{ Aged 20-64 y } & \multicolumn{2}{|l|}{ Aged $65-80$ y } \\
\hline & $\beta(95 \% \mathrm{Cl})$ & P-value & $\beta(95 \% \mathrm{Cl})$ & P-value & $\beta(95 \% \mathrm{Cl})$ & P-value & $\beta(95 \% \mathrm{Cl})$ & P-value \\
\hline \multicolumn{9}{|c|}{ Dominant hand grip strength $(\mathrm{kg})$} \\
\hline Age (y) & $-0.07(-0.09$ to -0.04$)$ & $<0.001$ & $-0.61(-0.70$ to -0.53$)$ & $<0.001$ & $-0.02(-0.04$ to -0.01$)$ & 0.003 & $-0.37(-0.43$ to -0.31$)$ & $<0.001$ \\
\hline $\mathrm{BMl}\left(\mathrm{kg} / \mathrm{m}^{2}\right)$ & 0.48 (0.39 to 0.57$)$ & $<0.001$ & 0.49 (0.32 to 0.65$)$ & $<0.001$ & 0.23 (0.17 to 0.28$)$ & $<0.001$ & 0.15 (0.04 to 0.25$)$ & 0.005 \\
\hline WC (cm) & $0.14(0.10$ to 0.17$)$ & $<0.001$ & 0.13 (0.08 to 0.19$)$ & $<0.001$ & 0.08 (0.06 to 0.10$)$ & $<0.001$ & $0.03(-0.01$ to 0.07$)$ & 0.151 \\
\hline $\mathrm{SBP}(\mathrm{mm} \mathrm{Hg})$ & 0.03 (0.01 to 0.05$)$ & 0.004 & $0.01(-0.02$ to 0.04$)$ & 0.591 & 0.01 (0.00 to 0.02$)$ & 0.251 & $0.00(-0.02$ to 0.02$)$ & 0.890 \\
\hline $\mathrm{DBP}(\mathrm{mm} \mathrm{Hg})$ & 0.06 (0.03 to 0.09$)$ & $<0.001$ & 0.15 (0.11 to 0.20$)$ & $<0.001$ & 0.03 (0.02 to 0.05$)$ & $<0.001$ & 0.05 (0.02 to 0.08$)$ & 0.002 \\
\hline $\mathrm{TG}(\mathrm{mg} / \mathrm{dL})$ & $0.00(0.00$ to 0.00$)$ & 0.014 & 0.00 (0.00 to 0.01$)$ & 0.012 & $0.00(0.00$ to 0.00$)$ & 0.752 & $0.00(-0.01$ to 0.00$)$ & 0.763 \\
\hline $\mathrm{HDL}(\mathrm{mg} / \mathrm{dL})$ & $-0.02(-0.04$ to 0.01$)$ & 0.264 & 0.04 (0.00 to 0.09$)$ & 0.054 & $-0.01(-0.03$ to 0.00$)$ & 0.061 & 0.04 (0.01 to 0.07$)$ & 0.003 \\
\hline LDL (mg/dL) & 0.01 (0.00 to 0.02$)$ & 0.123 & 0.02 (0.00 to 0.03$)$ & 0.008 & $0.00(-0.01$ to 0.00$)$ & 0.543 & 0.01 (0.00 to 0.02 ) & 0.092 \\
\hline Glucose (mg/dL) & $-0.01(-0.02$ to 0.00$)$ & 0.082 & $0.01(-0.01$ to 0.02$)$ & 0.369 & 0.00 (-0.01 to 0.02$)$ & 0.455 & $0.00(-0.02$ to 0.01$)$ & 0.504 \\
\hline $\mathrm{HbA1C}(\%)$ & $-0.27(-0.59$ to 0.05$)$ & 0.095 & $-0.06(-0.55$ to 0.42$)$ & 0.794 & $-0.08(-0.40$ to 0.23$)$ & 0.618 & $-0.25(-0.62$ to & 0.189 \\
\hline hsCRP (mg/L) & $-0.17(-0.29$ to -0.06$)$ & 0.004 & $-0.20(-0.35$ to -0.05$)$ & 0.010 & $0.04(-0.04$ to 0.12$)$ & 0.283 & $-0.16(-0.29$ to -0.04$)$ & 0.011 \\
\hline $\begin{array}{l}\text { Physical activity } \\
\text { (min/wk) }\end{array}$ & 0.01 (0.00 to 0.01$)$ & $<0.001$ & 0.01 (0.01 to 0.02 ) & $<0.001$ & 0.01 (0.00 to 0.01$)$ & 0.005 & 0.01 (0.00 to 0.02 ) & 0.002 \\
\hline Diabetes & $-1.54(-2.46$ to -0.61$)$ & 0.001 & $03(-1)$. & 0.954 & $-0.64(-1$. & 0.078 & $-0.99(-1.78$ to -0.19$)$ & 0.016 \\
\hline Dyslipidemia & 1.06 (0.42 to 1.70$)$ & 0.001 & 1.49 (0.51 to 2.47 ) & 0.003 & $0.03(-0.32$ & 0.871 & 0 2.24) & 0.001 \\
\hline Smoking & 0.83 (0.24 to 1.41$)$ & 0.006 & $0.00(-1.38$ to 1.38$)$ & & 0.89 (0.02 to 1.76$)$ & 0.045 & $-0.94(-3.20$ to 1.32$)$ & 0.414 \\
\hline Drinking & 1.75 (1.07 to & $<0.001$ & 1.5 & 0.004 & 0671 & $<0.001$ & 090 & 0.042 \\
\hline \multicolumn{9}{|c|}{ Relative hand grip strength (kg/BMI) } \\
\hline Age $(y)$ & $-0.01(-0.01$ & $<0$ & 4) & & & & 02) & $<0.001$ \\
\hline BMI $\left(\mathrm{kg} / \mathrm{m}^{2}\right)$ & $-0.09(-0.10$ to -0.09$)$ & $<0.001$ & $-0.08(-0.09$ to -0.06$)$ & $<0.001$ & $-0.07(-0.07$ to -0.07$)$ & $<0.001$ & $-0.05(-0.06$ to -0.04$)$ & $<0.001$ \\
\hline WC (cm) & $-0.03(-0.04$ to -0.03$)$ & $<0.001$ & $-0.02(-0.03$ to -0.02$)$ & $<0.001$ & $-0.02(-0.02$ to -0.02$)$ & $<0.001$ & $-0.02(-0.02$ to -0.01$)$ & $<0.001$ \\
\hline $\mathrm{SBP}(\mathrm{mm} \mathrm{Hg})$ & $-0.01(-0.01$ to -0.01$)$ & $<0.001$ & $0.00(0.00$ to 0.00$)$ & 0.623 & $-0.01(-0.01$ to -0.01$)$ & $<0.001$ & $0.00(0.00$ to 0.00$)$ & 0.996 \\
\hline $\mathrm{DBP}(\mathrm{mm} \mathrm{Hg})$ & $-0.01(-0.01$ to -0.01$)$ & $<0.001$ & 0.01 (0.01 to 0.01$)$ & $<0.001$ & $-0.01(-0.01$ to -0.01$)$ & $<0.001$ & 0.00 (0.00 to 0.01$)$ & 0.040 \\
\hline $\mathrm{TG}(\mathrm{mg} / \mathrm{dL})$ & $0.00(0.00$ to 0.00$)$ & $<0.001$ & $0.00(0.00$ to 0.00$)$ & & $0.00(0.00$ to 0.00$)$ & $<0.001$ & $0.00(0.00$ to 0.00$)$ & 0.005 \\
\hline $\mathrm{HDL}(\mathrm{mg} / \mathrm{dL})$ & 0.01 (0.01 to 0.01$)$ & $<0.001$ & 0.01 (0.01 to 0.01$)$ & $<0.001$ & 0.01 (0.01 to 0.01$)$ & $<0.001$ & $0.00(0.00$ to 0.01$)$ & $<0.001$ \\
\hline LDL (mg/dL) & $0.00(0.00$ to 0.00$)$ & 0.007 & $0.00(0.00$ to 0.00$)$ & 0.006 & $0.00(0.00$ to 0.00$)$ & $<0.001$ & $0.00(0.00$ to 0.00$)$ & 0.030 \\
\hline $\begin{array}{l}\text { Fasting glucose } \\
\text { (mg/dL) }\end{array}$ & $0.00(-0.01$ to 0.00$)$ & $<0.001$ & $0.00(0.00$ to 0.00$)$ & 0.204 & $0.00(-0.01$ to 0.00$)$ & $<0.001$ & 0.00 (0.00 to 0.00$)$ & 0.028 \\
\hline $\mathrm{HbA1C}(\%)$ & $-0.11(-0.14$ to -0.08$)$ & $<0.001$ & $-0.06(-0.11$ to -0.01$)$ & 0.011 & $-0.14(-0.17$ to -0.12$)$ & $<0.001$ & $-0.05(-0.08$ to -0.01$)$ & 0.004 \\
\hline hsCRP (mg/L) & $-0.04(-0.05$ to -0.03$)$ & $<0.001$ & $-0.01(-0.03$ to 0.00$)$ & 0.059 & $-0.04(-0.05$ to -0.03$)$ & $<0.001$ & $-0.01(-0.02$ to 0.00$)$ & 0.012 \\
\hline $\begin{array}{l}\text { Physical activity } \\
\text { (min/wk) }\end{array}$ & 0.00 (0.00 to 0.00$)$ & 0.001 & 0.00 (0.00 to 0.00$)$ & 0.007 & 0.00 (0.00 to 0.00$)$ & 0.001 & 0.00 (0.00 to 0.00$)$ & $<0.001$ \\
\hline Diabetes & $-0.33(-0.40$ to -0.26$)$ & $<0.001$ & $-0.13(-0.23$ to -0.02$)$ & 0.019 & $-0.34(-0.40$ to -0.28$)$ & $<0.001$ & $-0.12(-0.19$ to -0.04$)$ & 0.002 \\
\hline Dyslipidemia & $-0.19(-0.24$ to -0.13$)$ & $<0.001$ & 0.00 (-0.08 to 0.09$)$ & 0.955 & $-0.18(-0.21$ to -0.15$)$ & $<0.001$ & $0.04(-0.03$ to 0.11$)$ & 0.277 \\
\hline Smoking & 0.09 (0.04 to 0.14$)$ & $<0.001$ & $0.10(-0.01$ to 0.20$)$ & 0.077 & $0.02(-0.06$ to 0.10$)$ & 0.610 & $-0.08(-0.23$ to 0.07$)$ & 0.281 \\
\hline Drinking & 0.17 (0.11 to 0.23$)$ & $<0.001$ & 0.12 (0.02 to 0.21$)$ & 0.013 & 0.07 (0.04 to 0.10$)$ & $<0.001$ & 0.07 (0.00 to 0.14$)$ & 0.049 \\
\hline
\end{tabular}

Values are from linear regression analysis; coefficients are unstandardized.

BMI, body mass index; Cl, confidence interval; WC, waist circumference; SBP, systolic blood pressure; DBP, diastolic blood pressure; TG, triglycerides; HDL, high-density lipoprotein; LDL, low-density lipoprotein; HbA1C, hemoglobin A1c; hsCRP, high-sensitivity C-reactive protein. 
Table 3. Results from multivariate linear regression analysis of the associations of relative handgrip strength (strength/BMl) and dominant handgrip strength (kg) with cardiometabolic markers

\begin{tabular}{|c|c|c|c|c|c|c|c|c|}
\hline \multirow{3}{*}{ Predictor } & \multicolumn{4}{|c|}{ Men } & \multicolumn{4}{|c|}{ Women } \\
\hline & \multicolumn{2}{|l|}{ Aged 20-64 y } & \multicolumn{2}{|l|}{ Aged $65-80$ y } & \multicolumn{2}{|l|}{ Aged 20-64 y } & \multicolumn{2}{|l|}{ Aged 65-80 y } \\
\hline & $\beta(95 \% \mathrm{Cl})$ & P-value & $\beta(95 \% \mathrm{Cl})$ & P-value & $\beta(95 \% \mathrm{Cl})$ & P-value & $\beta(95 \% \mathrm{Cl})$ & P-value \\
\hline \multicolumn{9}{|c|}{ Dominant hand grip strength (kg) } \\
\hline BMl $\left(\mathrm{kg} / \mathrm{m}^{2}\right)$ & 0.65 (0.46 to 0.85$)$ & $<0.001$ & $0.21(-0.13$ to 0.56$)$ & 0.218 & $0.18(0.07$ to 0.29$)$ & 0.002 & $0.10(-0.07$ to 0.27$)$ & 0.241 \\
\hline WC (cm) & $-0.07(-0.14$ to 0.00$)$ & 0.062 & 0.06 (-0.05 to 0.17$)$ & 0.263 & $0.04(0.00$ to 0.09$)$ & 0.045 & $0.02(-0.05$ to 0.08$)$ & 0.613 \\
\hline $\mathrm{SBP}(\mathrm{mm} \mathrm{Hg})$ & $-0.01(-0.04$ to 0.02$)$ & 0.614 & $-0.01(-0.03$ to 0.02$)$ & 0.633 & $-0.03(-0.05$ to 0.00$)$ & 0.019 & $0.01(-0.01$ to 0.03$)$ & 0.430 \\
\hline $\mathrm{DBP}(\mathrm{mm} \mathrm{Hg})$ & $0.03(-0.01$ to 0.08$)$ & 0.118 & $0.06(0.00$ to 0.12$)$ & 0.042 & 0.05 (0.02 to 0.08$)$ & 0.001 & $0.01(-0.03$ to 0.05$)$ & 0.639 \\
\hline $\mathrm{TG}(\mathrm{mg} / \mathrm{dL})$ & $0.00(0.00$ to 0.00$)$ & 0.402 & 0.00 (0.00 to 0.01$)$ & 0.365 & $0.00(-0.01$ to 0.00$)$ & 0.020 & $0.00(-0.01$ to 0.00$)$ & 0.396 \\
\hline $\mathrm{HDL}(\mathrm{mg} / \mathrm{dL})$ & 0.01 (-0.02 to 0.04$)$ & 0.485 & 0.06 (0.02 to 0.09$)$ & 0.005 & $-0.01(-0.03$ to 0.00$)$ & 0.187 & $0.02(-0.01$ to 0.04$)$ & 0.248 \\
\hline LDL (mg/dL) & 0.00 (-0.01 to 0.01$)$ & 0.903 & 0.01 (0.00 to 0.02$)$ & 0.079 & $-0.01(-0.01$ to 0.00$)$ & 0.118 & $0.00(-0.01$ to 0.01$)$ & 0.672 \\
\hline $\begin{array}{l}\text { Fasting glucose } \\
(\mathrm{mg} / \mathrm{dL})\end{array}$ & $-0.01(-0.03$ to 0.02$)$ & 0.604 & 0.01 (-0.01 to 0.04$)$ & 0.418 & 0.02 (0.00 to 0.03$)$ & 0.112 & $0.01(-0.01$ to 0.03$)$ & 0.178 \\
\hline $\mathrm{HbA1C}(\%)$ & 0.18 (-0.40 to 0.75$)$ & 0.544 & $-0.68(-1.45$ to 0.10$)$ & 0.086 & $-0.31(-0.82$ to 0.19$)$ & 0.225 & $0.02(-0.57$ to 0.61$)$ & 0.951 \\
\hline hsCRP (mg/L) & $-0.19(-0.29$ to -0.08$)$ & $<0.001$ & $-0.12(-0.25$ to 0.01$)$ & 0.069 & $-0.09(-0.17$ to -0.01$)$ & 0.028 & $-0.14(-0.27$ to 0.00$)$ & 0.045 \\
\hline $\begin{array}{l}\text { Physical activity } \\
\text { (min/wk) }\end{array}$ & 0.01 (0.00 to 0.01$)$ & $<0.001$ & 0.01 (0.00 to 0.01 ) & 0.022 & 0.01 (0.00 to 0.01$)$ & 0.009 & 0.00 (0.00 to 0.01$)$ & 0.471 \\
\hline Diabetes & $-1.37(-2.72$ to -0.02$)$ & 0.046 & $0.22(-1.15$ to 1.59$)$ & 0.754 & $-1.38(-2.48$ to -0.27$)$ & 0.015 & $-1.23(-2.36$ to -0.09$)$ & 0.035 \\
\hline Dyslipidemia & $0.43(-0.28$ to 1.15$)$ & 0.235 & $0.22(-0.80$ to 1.24$)$ & 0.668 & $0.24(-0.20$ to 0.68$)$ & 0.284 & $0.68(-0.21$ to 1.58$)$ & 0.136 \\
\hline $\mathrm{R}^{2}$ & 0.10 & & 0.25 & & 0.06 & & 0.17 & \\
\hline \multicolumn{9}{|c|}{ Relative hand grip strength (kg/BMl) } \\
\hline BMI $\left(\mathrm{kg} / \mathrm{m}^{2}\right)$ & $-0.08(-0.09$ to -0.06$)$ & $<0.001$ & $-0.10(-0.12$ to -0.07$)$ & $<0.001$ & $-0.07(-0.08$ to -0.06$)$ & $<0.001$ & $-0.06(-0.07$ to -0.04$)$ & $<0.001$ \\
\hline WC (cm) & $-0.01(-0.01$ to 0.00$)$ & 0.073 & 0.01 (0.00 to 0.01$)$ & 0.230 & 0.00 (0.00 to 0.01$)$ & 0.080 & 0.00 (0.00 to 0.01$)$ & 0.598 \\
\hline $\mathrm{SBP}(\mathrm{mm} \mathrm{Hg})$ & $0.00(0.00$ to 0.00$)$ & 0.509 & $0.00(0.00$ to 0.00$)$ & 0.952 & $0.00(0.00$ to 0.00$)$ & 0.015 & $0.00(0.00$ to 0.00$)$ & 0.398 \\
\hline $\mathrm{DBP}(\mathrm{mm} \mathrm{Hg})$ & $0.00(0.00$ to 0.01$)$ & 0.065 & 0.01 (0.00 to 0.01$)$ & 0.024 & $0.00(0.00$ to 0.01$)$ & $<0.001$ & $0.00(0.00$ to 0.00$)$ & 0.526 \\
\hline $\mathrm{TG}(\mathrm{mg} / \mathrm{dL})$ & $0.00(0.00$ to 0.00$)$ & 0.519 & $0.00(0.00$ to 0.00$)$ & 0.408 & $0.00(0.00$ to 0.00$)$ & 0.009 & $0.00(0.00$ to 0.00$)$ & 0.111 \\
\hline $\mathrm{HDL}(\mathrm{mg} / \mathrm{dL})$ & $0.00(0.00$ to 0.00$)$ & 0.052 & 0.00 (0.00 to 0.01$)$ & 0.003 & $0.00(0.00$ to 0.00$)$ & 0.634 & $0.00(0.00$ to 0.00$)$ & 0.683 \\
\hline LDL (mg/dL) & $0.00(0.00$ to 0.00$)$ & 0.815 & $0.00(0.00$ to 0.00$)$ & 0.149 & $0.00(0.00$ to 0.00$)$ & 0.017 & $0.00(0.00$ to 0.00$)$ & 0.959 \\
\hline $\begin{array}{l}\text { Fasting glucose } \\
(\mathrm{mg} / \mathrm{dL})\end{array}$ & $0.00(0.00$ to 0.00$)$ & 0.276 & 0.00 (0.00 to 0.00$)$ & 0.197 & 0.00 (0.00 to 0.00$)$ & 0.085 & $0.00(0.00$ to 0.00$)$ & 0.114 \\
\hline $\mathrm{HbA1C}(\%)$ & 0.03 (-0.01 to 0.07$)$ & 0.175 & $-0.06(-0.12$ to 0.00$)$ & 0.067 & $-0.02(-0.06$ to 0.02$)$ & 0.327 & $-0.01(-0.06$ to 0.03$)$ & 0.600 \\
\hline hsCRP (mg/L) & $-0.01(-0.02$ to -0.01$)$ & $<0.001$ & $-0.01(-0.02$ to 0.00$)$ & 0.122 & $-0.01(-0.01$ to 0.00$)$ & 0.034 & $-0.01(-0.02$ to 0.00$)$ & 0.029 \\
\hline $\begin{array}{l}\text { Physical activity } \\
\text { (min/wk) }\end{array}$ & $0.00(0.00$ to 0.00$)$ & $<0.001$ & $0.00(0.00$ to 0.00$)$ & 0.009 & $0.00(0.00$ to 0.00$)$ & 0.011 & $0.00(0.00$ to 0.00$)$ & 0.126 \\
\hline Diabetes & $-0.12(-0.22$ to -0.02$)$ & 0.017 & $-0.03(-0.14$ to 0.08$)$ & 0.595 & $-0.10(-0.18$ to -0.02$)$ & 0.020 & $-0.08(-0.17$ to 0.01$)$ & 0.085 \\
\hline Dyslipidemia & $0.01(-0.05$ to 0.06$)$ & 0.847 & $0.00(-0.09$ to 0.08$)$ & 0.950 & $0.01(-0.02$ to 0.05$)$ & 0.428 & $0.06(-0.01$ to 0.14$)$ & 0.083 \\
\hline $\mathrm{R}^{2}$ & 0.31 & & 0.35 & & 0.33 & & 0.30 & \\
\hline
\end{tabular}

Values are from linear regression analysis; coefficients are unstandardized. Age was adjusted for in the multivariate linear model.

BMI, body mass index; $\mathrm{Cl}$, confidence interval; WC, waist circumference; SBP, systolic blood pressure; DBP, diastolic blood pressure; TG, triglycerides; HDL, high-density lipoprotein; LDL, low-density lipoprotein; HbA1C, hemoglobin A1c; hsCRP, high-sensitivity C-reactive protein.

had a variance inflation factor of $>10$, from regression analysis. The results of univariate and multivariate regression analyses are shown in Tables 2 and 3, respectively.

In male participants aged 20-64 years, dominant HGS showed a positive relationship to BMI and physical activity. Relative HGS was positively associated with BMI and physical activity. Both relative and dominant HGS showed a negative association with hsCRP and diabetes.

In male participants aged 65-80 years, dominant HGS showed a positive correlation with HDL. Relative HGS showed a negative correlation with BMI. Both dominant and relative HGS were positively associated with diastolic blood pressure. Physical activity duration showed a positive relationship to HGS in men, regardless of age group or HGS type.
In female participants aged 20-64 years, dominant HGS showed a significantly positive correlation with BMI and waist circumference, whereas it showed a negative relation to systolic blood pressure. Relative HGS showed a significantly positive correlation with systolic blood pressure and LDL, whereas it was negatively associated with BMI. In both HGS groups, hsCRP and diabetes showed a negative correlation with HGS. Triglyceride, diastolic blood pressure, and physical activity duration showed a positive correlation with HGS.

In female participants aged 65-80 years, dominant HGS showed a significantly negative correlation with diabetes. Relative HGS was inversely associated with BMI. hsCRP showed a significantly negative association with HGS in both grip strength groups. Dyslipidemia did not show any significant association with HGS. 


\section{DISCUSSION}

For many years, researchers have studied the importance of sarcopenia and its relation to health and aging. In this study, we examined the associations between HGS and cardiometabolic markers in the Korean adult population. Our results showed that HGS is inversely associated with hsCRP in women and younger men, and is inversely related to diabetes in the younger group.

\section{Grip Strength and Obesity}

In this study, dominant HGS showed a positive association with BMI in men and women aged 20-64 years, which was consistent with the result of a previous study, ${ }^{4)}$ whereas relative HGS showed a negative association with BMI in all sex and age groups. Relative HGS refers to absolute HGS divided by BMI. Larger muscle mass could contribute to larger BMI, which could decrease the relative HGS. This seems to be a mathematical phenomenon.

In a cross-sectional study of strength index, body weight was similar among strength quintiles, although an inverse relationship was observed between muscular strength and the prevalence and incidence of excessive body fat and excessive abdominal fat. ${ }^{20)}$ Another study on associations between resistance training and body composition showed that resistance training seems to have positive effects on body composition without significant effects on weight decrease. ${ }^{21)}$ People aged 20-64 years tend to maintain physical activity and muscle mass compared with older people. Active physical movements could maintain the lean body mass, which could decrease the percentage body fat but not the total fat mass or absolute body weight.

\section{Grip Strength and Blood Pressure}

In our study, higher relative and absolute HGS values were negatively related to systolic blood pressure in women aged 20-64 years. Higher diastolic blood pressure showed a positive association with relative HGS in men and younger women aged 20-64 years. Several studies showed conflicting results with respect to blood pressure and HGS.

In one cross-sectional study assessing the association between HGS and blood pressure in middle-aged (mean, 63.2 \pm 6.6 years) and oldest old (age $\geq 85$ years) participants, HGS was positively associated with systolic blood pressure only in the oldest old group, whereas there was no significant association in the middle-aged group. The results suggested that higher blood pressure is positively associated with better muscle strength in old age. ${ }^{22)}$ In a Chinese study on adolescents aged 13-17 years, increased BMI was associated with high blood pressure and low HGS. However, after adjustment for BMI, high HGS showed a positive relationship to blood pressure. ${ }^{23)}$ These two studies showed different results from those of the present study owing to the analysis of different age groups.

In another study including 4,846 participants older than 60 years, participants with sarcopenia had a higher prevalence of hypertension than those without sarcopenia. A strong association between hypertension and sarcopenia was observed in patients with diabetes melli- tus. A total of 876 patients (18.1\%) with diabetes and 3,970 patients $(81.9 \%)$ without diabetes were included. ${ }^{24)}$ This study included a higher number of healthy participants than that in our study. Our study included $32.3 \%$ participants with impaired fasting glucose. To confirm the relationship between blood pressure and muscle mass, further studies adjusting for diabetes are needed.

\section{Grip Strength and Inflammation (High-Sensitivity C-Reactive Protein)}

Previous studies have shown that hsCRP is related to cardiovascular diseases. ${ }^{25,26)}$ Schaap et al. ${ }^{27)}$ showed that higher levels of interleukin-6 and hsCRP are related to a high risk of muscle strength loss. This study showed negative associations of hsCRP with relative HGS in younger men and in all women, consistent with previous studies. A Japanese study reported that hsCRP is inversely associated with HGS in the elderly. ${ }^{6)}$ Another cross-sectional study on older populations from several countries showed similar results in univariate analysis. After adjustment for socioeconomic factors and health behaviors, the significant association disappeared. ${ }^{7)}$ Further studies are needed to discuss the discrepancy of our results with previous results in older men.

No strong associations were found between HGS and lipid profile. The results were partly consistent with those of a previous cross-sectional studies. ${ }^{5,8)}$

\section{Grip Strength and Diabetes}

In our study, diabetes was inversely related to both types of HGS in younger participants of both sexes. The results were consistent with those of a study on six ethnic groups, which found an inverse relationship between HGS and the prevalence of type 2 diabetes mellitus, regardless of grip strength differences. ${ }^{28)}$ In another study from China, the third and fourth quartiles of relative HGS were negatively related to impaired fasting glucose in male adults. ${ }^{10)}$ Conversely, the risk of type 2 diabetes mellitus did not show an association with grip strength in participants aged 50-75 years in a prospective cohort study. ${ }^{29)}$ A study using data from KNHANES 2014-2015 reported that HGS is inversely associated with fasting glucose, $\mathrm{HbAlC}$, fasting insulin, and some calculated levels [\{fasting glucose $(\mathrm{mg} / \mathrm{dL}) \times$ fasting insulin $(\mathrm{mIU} / \mathrm{L})\} / 40]$. The percentage mediation effect of hsCRP ranged from $8 \%$ to $11 \% .{ }^{15}$ This previous study showed some differences from our study despite similarly using data from KNHANES. First, our study used more recent data from 2015 to 2016. Second, the previous authors did not stratify the participants according to sex and age, despite the different HGS between the sexes (mean HGS, 39.67; 95\% CI, 39.32-40.02 and mean HGS, 23.70; 95\% CI, 23.48-23.91 for men and women aged between 19 and 80 years, respectively). We divided the participants into groups according to sex and age, and different outcomes were obtained among them. Third, Lee et al. ${ }^{15)}$ employed the protocol of the American Society of Hand Therapists to measure HGS, which uses the mean HGS from three trials. On the contrary, our study employed the Southampton protocol, which uses the maximal grip score from three trials. Grip strength measurements could be affected by the protocol used. After 
multiple attempts, strength could become lower and the maximum grip strength may be greater than the mean value obtained after three trials. Therefore, we used the Southampton protocol to measure HGS according to the suggestion by Roberts et al. ${ }^{17)}$ Fourth, our study included blood pressure and dyslipidemia, which were not included in a study of Lee et al. ${ }^{15)}$ Our analysis showed that blood pressure and lipid profile were also associated with HGS in some groups (Table 3). In the future, studies including grip strength stratification and older-aged participants may be necessary.

Our study had some strengths. First, this study evaluated the relationship of HGS to variable cardiovascular markers using data from a well-conducted nationally representative survey in South Korea. Second, to our knowledge, this study is the first to assess the association between two kinds of HGS, relative and dominant, and cardiometabolic outcomes in stratified sex and age groups in the Korean adult population. Our study revealed that HGS has negative a relationship to BMI, hsCRP, and diabetes, and a positive relationship to physical activity in both sexes in younger age. However, those relationships were reduced to nonsignificance after old age, in which the associations between dominant HGS and BMI, relative HGS and hsCRP, and relative HGS and diabetes disappeared in both sexes. Unlike in men, HGS in young women showed some association with waist circumference, systolic blood pressure, diastolic blood pressure, and triglyceride level. However, those associations also disappeared in the analysis of old age.

\section{Grip Strength and Dyslipidemia}

In univariate linear regression, dominant HGS showed a significant positive association with dyslipidemia in men and older women aged 65-80 years. Relative HGS was positively associated with dyslipidemia in younger men and negatively associated with dyslipidemia in younger women. Our results were consistent with those from a recent study in China, in which only relative HGS in the higher quartiles was related to less risk of dyslipidemia and hyperlipidemia. ${ }^{10)}$ However, after multivariate adjustment in our study, these associations became insignificant. In one previous study, HGS showed moderate associations with lipid profiles and other cardiometabolic factors. ${ }^{4)}$ Further longitudinal studies would be needed to explore the role of HGS in dyslipidemia.

\section{Limitations}

Our study had several limitations. First, as this study had a cross-sectional design, the existence of a causal relationship between HGS and cardiometabolic markers could not be confirmed. Second, the analysis did not include variables of smoking, alcohol drinking, education, and other socioeconomic factors and health issues. Lastly, fat mass or muscle mass measurements for body composition were missing in this study.

Relative HGS has recently been suggested as a cardiometabolic predictor of muscle strength, showing a stronger association with cardiovascular disease markers than dominant HGS. ${ }^{8,9)}$ In our study, the relative HGS did not show a stronger association with CRP, blood pressure,
BMI, and physical activity duration than absolute HGS. However, relative HGS showed a positive association with HDL in men aged 20-64 years, whereas there was no such relationship for absolute HGS. This may be due to the minimization of the effect of body composition. Further studies are needed to estimate the compared odds ratios and relative risks.

\section{Conclusion}

Our study showed that increased HGS is associated with lower hsCRP in women and younger men, and is related to a lower prevalence of diabetes in younger people. HGS is a quick and simple method of measuring muscle strength. This suggests that HGS may be an effective screening tool for cardiometabolic disease. Further studies, including prospective cohort studies, may be needed in the older and adolescent populations.

\section{CONFLICT OF INTEREST}

No potential conflict of interest relevant to this article was reported.

\section{ORCID}

Hanul Chong: https://orcid.org/0000-0002-7978-4973

Young Eun Choi: https://orcid.org/0000-0002-7657-7776

Jin Young Kong: https://orcid.org/0000-0002-5612-987X

Joo Hyun Park: https://orcid.org/0000-0002-9980-4222

Hyun Jeong Yoo: https://orcid.org/0000-0002-3391-6459

Jeong Ho Byeon: https://orcid.org/0000-0002-2219-1982

Hye Jun Lee: https://orcid.org/0000-0001-5810-9787

Sang Hyun Lee: https://orcid.org/0000-0002-8847-183X

\section{REFERENCES}

1. Sayer AA, Syddall HE, Dennison EM, Martin HJ, Phillips DI, Cooper C, et al. Grip strength and the metabolic syndrome: findings from the Hertfordshire Cohort Study. QJM 2007;100:707-13.

2. Cetinus E, Buyukbese MA, Uzel M, Ekerbicer H, Karaoguz A. Hand grip strength in patients with type 2 diabetes mellitus. Diabetes Res Clin Pract 2005;70:278-86.

3. Ruiz JR, Sui X, Lobelo F, Morrow JR Jr, Jackson AW, Sjostrom M, et al. Association between muscular strength and mortality in men: prospective cohort study. BMJ 2008;337:a439.

4. Gubelmann C, Vollenweider P, Marques-Vidal P. Association of grip strength with cardiovascular risk markers. Eur J Prev Cardiol 2017;24: 514-21.

5. Yamada E, Takeuchi M, Kurata M, Tsuboi A, Kazumi T, Fukuo K. Low haemoglobin levels contribute to low grip strength independent of low-grade inflammation in Japanese elderly women. Asia Pac J Clin Nutr 2015;24:444-51.

6. Yoshida Y, Iwasa H, Kumagai S, Yoshida H, Suzuki T. Association between C-reactive protein (CRP) level and physical performance in community-dwelling elderly in Japan. Arch Gerontol Geriatr 2010;51: 
164-8.

7. Sousa AC, Zunzunegui MV, Li A, Phillips SP, Guralnik JM, Guerra RO. Association between C-reactive protein and physical performance in older populations: results from the International Mobility in Aging Study (IMIAS). Age Ageing 2016;45:274-80.

8. Lawman HG, Troiano RP, Perna FM, Wang CY, Fryar CD, Ogden CL. Associations of relative handgrip strength and cardiovascular disease biomarkers in U.S. adults, 2011-2012. Am J Prev Med 2016;50:677-83.

9. Lee WJ, Peng LN, Chiou ST, Chen LK. Relative handgrip strength is a simple indicator of cardiometabolic risk among middle-aged and older people: a nationwide population-based study in Taiwan. PLoS One 2016;11:e0160876.

10. Li D, Guo G, Xia L, Yang X, Zhang B, Liu F, et al. Relative handgrip strength is inversely associated with metabolic profile and metabolic disease in the general population in China. Front Physiol 2018;9:59.

11. Chin SO, Rhee SY, Chon S, Hwang YC, Jeong IK, Oh S, et al. Sarcopenia is independently associated with cardiovascular disease in older Korean adults: the Korea National Health and Nutrition Examination Survey (KNHANES) from 2009. PLoS One 2013;8:e60119.

12. Yang EJ, Lim S, Lim JY, Kim KW, Jang HC, Paik NJ. Association between muscle strength and metabolic syndrome in older Korean men and women: the Korean Longitudinal Study on Health and Aging. Metabolism 2012;61:317-24.

13. Yi DW, Khang AR, Lee HW, Son SM, Kang YH. Relative handgrip strength as a marker of metabolic syndrome: the Korea National Health and Nutrition Examination Survey (KNHANES) VI (20142015). Diabetes Metab Syndr Obes 2018;11:227-40.

14. Oh YH, Moon JH, Kong MH, Oh B, Kim HJ. The association between hand grip strength and health-related quality of life in Korean adults. Korean J Sports Med 2017;35:103-11.

15. Lee MR, Jung SM, Bang H, Kim HS, Kim YB. Association between muscle strength and type 2 diabetes mellitus in adults in Korea: data from the Korea National Health and Nutrition Examination Survey (KNHANES) VI. Medicine (Baltimore) 2018;97:e10984.

16. Yoo JI, Choi H, Ha YC. Mean hand grip strength and cut-off value for sarcopenia in Korean adults using KNHANES VI. J Korean Med Sci 2017;32:868-72.

17. Roberts HC, Denison HJ, Martin HJ, Patel HP, Syddall H, Cooper C, et al. A review of the measurement of grip strength in clinical and epidemiological studies: towards a standardised approach. Age Ageing 2011;40:423-9.
18. Choquette S, Bouchard DR, Doyon CY, Senechal M, Brochu M, Dionne IJ. Relative strength as a determinant of mobility in elders 67-84 years of age: a NuAge Study: nutrition as a determinant of successful aging. J Nutr Health Aging 2010;14:190-5.

19. American Diabetes Association. 2. Classification and diagnosis of diabetes: standards of medical care in diabetes-2018. Diabetes Care 2018; 41:S13-27.

20. Jackson AW, Lee DC, Sui X, Morrow JR Jr, Church TS, Maslow AL, et al. Muscular strength is inversely related to prevalence and incidence of obesity in adult men. Obesity (Silver Spring) 2010;18:1988-95.

21. Donnelly JE, Smith B, Jacobsen DJ, Kirk E, Dubose K, Hyder M, et al. The role of exercise for weight loss and maintenance. Best Pract Res Clin Gastroenterol 2004;18:1009-29.

22. Taekema DG, Maier AB, Westendorp RG, de Craen AJ. Higher blood pressure is associated with higher handgrip strength in the oldest old. Am J Hypertens 2011;24:83-9.

23. Dong B, Wang Z, Arnold L, Song Y, Wang HJ, Ma J. The association between blood pressure and grip strength in adolescents: does body mass index matter? Hypertens Res 2016;39:919-25.

24. Han K, Park YM, Kwon HS, Ko SH, Lee SH, Yim HW, et al. Sarcopenia as a determinant of blood pressure in older Koreans: findings from the Korea National Health and Nutrition Examination Surveys (KNHANES) 2008-2010. PLoS One 2014;9:e86902.

25. Cesari M, Penninx BW, Newman AB, Kritchevsky SB, Nicklas BJ, Sutton-Tyrrell $\mathrm{K}$, et al. Inflammatory markers and cardiovascular disease (the Health, Aging and Body Composition [Health ABC] Study). Am J Cardiol 2003;92:522-8.

26. Vasan RS, Sullivan LM, Roubenoff R, Dinarello CA, Harris T, Benjamin EJ, et al. Inflammatory markers and risk of heart failure in elderly subjects without prior myocardial infarction: the Framingham Heart Study. Circulation 2003;107:1486-91.

27. Schaap LA, Pluijm SM, Deeg DJ, Visser M. Inflammatory markers and loss of muscle mass (sarcopenia) and strength. Am J Med 2006;119: 526.

28. Van der Kooi AL, Snijder MB, Peters RJ, van Valkengoed IG. The association of handgrip strength and type 2 diabetes mellitus in six ethnic groups: an analysis of the HELIUS study. PLoS One 2015;10:e0137739.

29. Marques-Vidal P, Vollenweider P, Waeber G, Jornayvaz FR. Grip strength is not associated with incident type 2 diabetes mellitus in healthy adults: the CoLaus study. Diabetes Res Clin Pract 2017;132: 144-8. 